\title{
Knockdown of Matrix Metallopeptidase 9 Inhibits Metastasis of Oral Squamous Cell Carcinoma Cells in a Zebrafish Xenograft Model
}

\author{
Jinlin Wen $\mathbb{D}^{1}{ }^{1}$ Panpan Yin, ${ }^{1}$ Linwei Li, ${ }^{2}$ Guihua Kang, ${ }^{2}$ Guozhu Ning, ${ }^{2}$ Yu Cao, ${ }^{2}$ Feng Gao, ${ }^{1}$ \\ Ying Su, ${ }^{1}$ Yanlin $W u,{ }^{1}$ and Xinyan Zhang ${ }^{1}$ \\ ${ }^{1}$ Beijing Institute of Dental Research, Beijing Stomatological Hospital \& School of Stomatology, Capital Medical University, \\ Beijing, China \\ ${ }^{2}$ State Key Laboratory of Membrane Biology, Institute of Zoology, University of Chinese Academy of Sciences, Chinese Academy \\ of Sciences, Beijing, China
}

Correspondence should be addressed to Xinyan Zhang; xinyanzhangzh@126.com

Received 5 December 2019; Revised 20 March 2020; Accepted 26 March 2020; Published 16 April 2020

Academic Editor: Gail Mahady

Copyright (c) 2020 Jinlin Wen et al. This is an open access article distributed under the Creative Commons Attribution License, which permits unrestricted use, distribution, and reproduction in any medium, provided the original work is properly cited.

\begin{abstract}
Destruction of extracellular matrix (ECM) is one of the basic steps of tumor invasion and metastasis. Matrix metalloproteinase (MMP) 9, a kind of zinc-ion-dependent endopeptidase, can degrade almost all protein components in the ECM, destroy the histological barrier of tumor cell invasion, and play a key role in tumor invasion and metastasis. The role of MMP-9 in tumor invasion and metastasis has attracted increasing attention and is considered the main proteolytic enzyme in this process. Although the overexpression of MMP-9 was detected in Oral squamous cell carcinoma (OSCC) tissues, further basic studies in vivo and in vitro are needed to investigate the role of MMP-9 in OSCCs and provide scientific validation. In this research, we developed a novel OSCC zebrafish xenograft model to study the role of the MMP-9 gene in oral carcinogenesis. Firstly, the MMP-9/shRNA lentiviral clone and control virus were constructed and transfected into OSCC cells. Then, the decreasing expression of MMP-9 was verified by RT-PCR and immunocytochemistry. Cell proliferation was detected by MTT assay. Colony formation was evaluated by colony formation assay. Cell invasion was evaluated using transwell invasion assay in vitro. In addition, OSCC cells with MMP-9/shRNA knockdown and control vector were injected into zebrafish and an OSCC tumor model in zebrafish was established to evaluate invasion and metastasis in vivo. Knockdown of MMP-9 gene by shRNA could inhibit OSCC cell growth and clone formation and markedly suppress cell invasion in vitro. And the knockdown of the MMP-9 gene could also significantly decrease the metastatic distance and number of metastatic tumor cells or lesions in vivo and suppress the metastasis rate in xenografted zebrafish. Taken together, these evidences indicated that the knockdown of MMP-9 might suppress OSCC cell invasion and metastasis in vivo and in vitro. The MMP-9 gene may be a promising therapeutic target for OSCCs in the future.
\end{abstract}

\section{Introduction}

Oral squamous cell carcinoma (OSCC) is one of most common types of head and neck cancer and about two-thirds of deaths are in developing countries [1]. Despite multidisciplinary treatment, the survival rate of patients is still only approximate 50\% [2]. Metastasis is one of the main causes of high mortality in OSCC patients. The OSCC cells tend to spread initially to regional lymph nodes in the neck before they spread to distant sites (lung or other organs) [3]. There- fore, inhibiting tumor metastasis is critical for improving clinical outcomes of OSCC patients.

Extracellular matrix (ECM), a highly dynamic noncellular component in tissue, can provide both biochemical and essential structural support for cellular components [4]. For cancers to commence invasion and metastasis, they must break the ECM barrier of the basement membrane [5]. Matrix metalloproteinases (MMPs), the family of calciumdependent and zinc-containing endopeptidases, can break the ECM barrier in physiological processes and in cancers 
[6]. Among all MMPs, MMP-9 is considered the especially compelling target and main proteolytic enzyme in this process, which regulates the pathological remodeling, degrades almost all protein components in the ECM, destroys the histological barrier of tumor cell invasion, and plays a central role in cancer invasion and metastasis [7]. In the previous studies, overexpression of MMP-9 has been reported to be associated with higher grade, metastasis, and angiogenesis in a large number of human cancers $[8,9]$. In particular, decreasing expression of MMP-9 could inhibit the invasive and metastatic ability of some cancers, such as pancreatic adenocarcinoma [10], lung cancer [11], thyroid cancer [12], and ovarian cancer [13]. But MMP-9 could also maintain the epithelial-mucosal integrity by activating the EGFR-Sp1 signaling pathway, thereby playing a suppressor role in colitis-associated cancer [14]. The overexpression of MMP9 in human OSCC tissues may contribute to cancer metastasis, suggesting that MMP-9 may serve as a prognostic biomarker of OSCC $[15,16]$. In addition, the elevated MMP-9 level in saliva may be a useful diagnostic factor for the early detection of OSCC $[17,18]$. Although the high expression of MMP-9 was considered to be associated with the invasion of OSCCs in clinical studies, further basic studies in vivo and in vitro are necessary to confirm the role of MMP-9 in OSCCs and provide scientific validation.

The zebrafish, first described by George Streisinger, emerged as an animal model of developmental genetics in the 1960s. Since then, zebrafish has been widely used in biomedical studies and has become an increasingly important cancer model [19]. Compared with other animal models, zebrafish provides a promising opportunity to monitor the dissemination and metastasis of single tumor cell owing to these advantages including large number of transparent eggs, rapid external development, immune-privileged feature, and less raised cost [20,21]. Recently, a series of zebrafish cancer models had been developed including uveal melanoma model [22], conjunctival melanoma model [23], glioma model [24], breast cancer model [25], malignant melanoma model [26], liposarcoma cancer model [27], and leukemia model [28]. Furthermore, zebrafish can absorb small molecular compounds from water and play an important role in efficiently and easily screening a large number of anticancer compounds [29]. In this study, we established a novel human OSCC model in zebrafish body to observe tumor progression in a real-time manner and confirm the possible function of MMP-9 in vivo. Our observations revealed that knockdown of MMP-9 might play the inhibitor role in the growth, invasion, and metastasis of OSCC cells in vivo and in vitro. Moreover, the MMP-9 gene may be a promising therapeutic target for OSCCs in the future.

\section{Materials and Methods}

2.1. Cell Line Culture. The OSCC cell lines CAL-27 and SCC15 were obtained from the Wuhan University and American Type Culture Collection (ATCC, Manassas, VA), respectively. The OSCC cells were routinely cultured in DMEM:F12 or DMEM high-glucose medium (Invitrogen Life Science, Carlsbad, CA) containing 10\% fetal bovine serum (FBS), $100 \mathrm{U} / \mathrm{ml}$ penicillin, and $100 \mu \mathrm{g} / \mathrm{ml}$ streptomycin with $5 \% \mathrm{CO}_{2}$ at $37^{\circ} \mathrm{C}$. All in vitro experiments were done in triplicate to ensure reliability.

2.2. Cell Line Transfection. The MMP-9/shRNA lentiviral clone and control shRNA vector were obtained from GeneCopoeia Inc. (Rockville, MD) (listed in Supplementary Figure 1). After confirming the knockdown of MMP-9, the most efficient shRNA sequence was selected for subsequent experiments. Then, the lentiviral knockdown plasmid using HIV, psi-LVRU6MP/MMP-9/shRNA, and Lenti-Pac HIV Expression Packaging Kit was cotransfected into 293Ta cells to package lentiviral particles and produce recombinant lentiviral particles. SCC-15 cells $\left(1.5 \times 10^{5} / \mathrm{ml}\right)$ and CAL-27 cells $\left(6 \times 10^{4} / \mathrm{ml}\right)$ were seeded into 6 -well plates one day before transfection. Then, the OSCC cell lines were transfected with lentiviruses containing MMP-9/shRNA. The lentivirus transfer vector expressing mCherry protein served as the control. To obtain stable lentiviral transduction of OSCC cell lines, we screened the OSCC cells with puromycin (Sigma-Aldrich, Saint Louis, MO). There was no significant change in the viability and growth of OSCC cells after labeling.

2.3. Real-Time PCR (RT-PCR) Analysis. The effect of decreasing MMP-9 expression at the RNA level was verified by RTPCR. Trizol reagent (ComWin Biotech Co., Ltd., CHN) was used to isolate the total RNA from transfected OSCC cells. Super RT cDNA Synthesis kit (ComWin Biotech) was used to get single-stranded cDNA. Then, ULtraSYBR Mixture kit (Low ROX) (ComWin Biotech) was used for quantitative PCR following the manufacturer's instructions. The PCR primer sets for human MMP-9 were provided by GeneCopoeia Inc. And GAPDH (forward, 5' -catgggtgtgaaccatgagaagtat- $3^{\prime}$; reverse, $5^{\prime}$-gactgtggtcatgagtccttcca- $3^{\prime}$ ) were provided from Sangon Biotech Co., Ltd. (CHN). The $\Delta \Delta \mathrm{Ct}$ method was used for quantification, and data were normalized to GAPDH (served as the endogenous control).

2.4. Immunocytochemistry. Reduced expression of MMP-9 at the protein level was detected by immunocytochemistry. The control vector cells and shRNA cells $\left(2 \times 10^{4} / \mathrm{ml}\right)$ were, respectively, seeded in 24 -well plates and cultured at $37^{\circ} \mathrm{C}$ with $5 \% \mathrm{CO}_{2}$. Then, cells were fixed with $10 \%$ neutral buffer formalin fixative for 10 mins and incubated with Triton X100 for 10 mins. And cells were blocked in PBS mixture containing $10 \%$ goat serum for $1 \mathrm{~h}$. Then, cells were incubated with mouse polyclonal anti-MMP-9 antibody (Proteintech Group, Inc., Rosemont, IL, $1: 1000)$ overnight at $4^{\circ} \mathrm{C}$. After cells were wasted with PBS, they were treated with hydrogen peroxide for 10 mins and stained with diaminobenzidine $(\mathrm{DAB})$ kit. The pictures were taken under an inverted microscope.

2.5. MTT Assay. The growth of OSCC cells were assessed by MTT assay. CAL-27/LVRU6MP (served as the control) and CAL-27/MMP-9/shRNA cells $\left(3 \times 10^{4} / \mathrm{ml}\right)$ and SCC15/LVRU6MP (served as the control) and SCC-15/MMP9/shRNA cells $\left(1 \times 10^{4} / \mathrm{ml}\right)$ were, respectively, seeded in 96- 

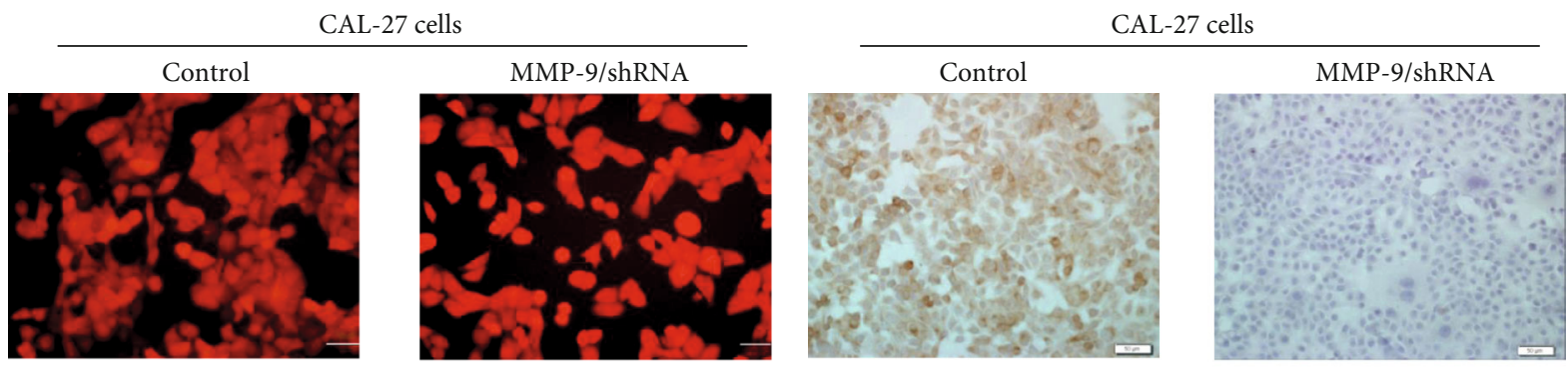

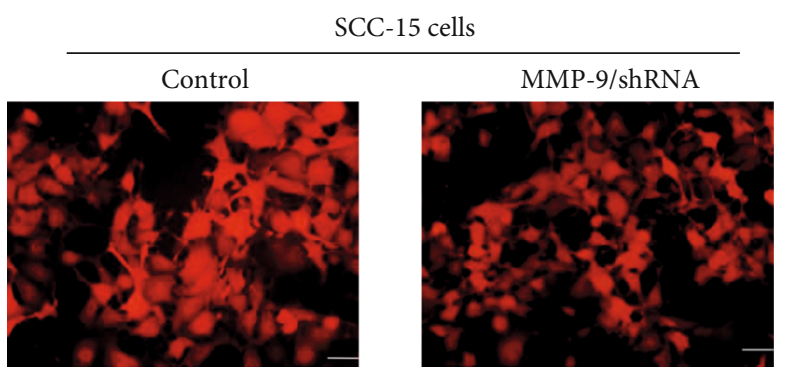

(a)

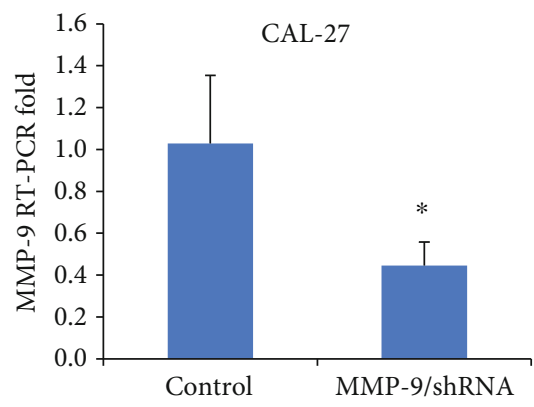

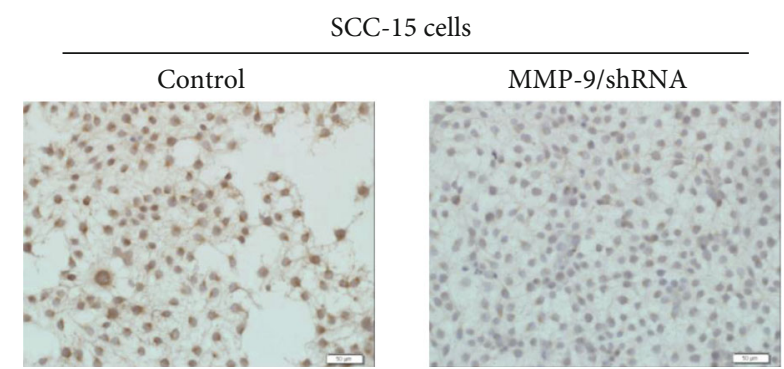

(b)

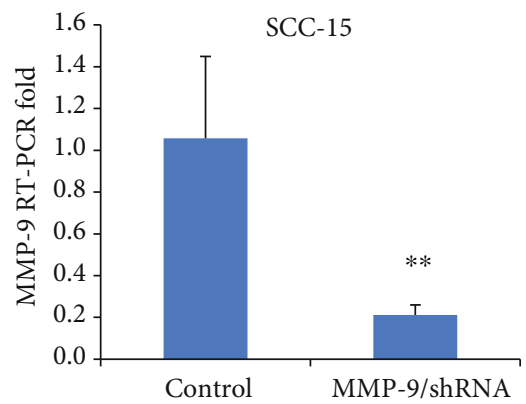

(c)

FIGURE 1: MMP-9 gene knockdown in OSCC cells. (a) The red fluorescence indicated the transfected cells (magnification, $\times 400$ ). (b) Compared with the control, MMP-9 expression at the protein level was reduced in MMP-9/shRNA groups by immunocytochemistry analysis. Bar $=50 \mu \mathrm{m}$. (c) The effect of decreasing MMP-9 expression at the RNA level was detected by RT-PCR. ${ }^{*} P<0.05$ and ${ }^{* *} P<0.01$ as compared with the control. $\mathrm{Bar}=200 \mathrm{~mm}$.

well plates and cultured for $24 \mathrm{~h}, 48 \mathrm{~h}$, and $72 \mathrm{~h}$ in an incubator with $5 \% \mathrm{CO}_{2}$. After adding $20 \mu \mathrm{l}$ MTT solution $(5 \mathrm{mg} / \mathrm{ml})$ in 96-well plates, the cells were incubated for $4 \mathrm{~h}$. To dissolve reactant, $200 \mu$ l dimethyl sulfoxide (DMSO, Sigma-Aldrich Corp) was added to each well. Then, these plates were read at a wavelength of $490 \mathrm{~nm}$ using a microplate reader (Sunnyvale, $C A$ ), and the optical density (OD) value was measured.

2.6. Colony Formation Assay. The colony formation ability of OSCC cells was evaluated by colony formation assay. The $100 \mu \mathrm{l} \mathrm{CAL-27/LVRU6MP} \mathrm{and} \mathrm{CAL-27/MMP-9/shRNA} \mathrm{cell}$ $\left(1 \times 10^{4} / \mathrm{ml}\right)$ and SCC-15/LVRU6MP and SCC-15/MMP9/shRNA cell $\left(5 \times 10^{3} / \mathrm{ml}\right)$ suspensions were, respectively, prepared and seeded in $60 \mathrm{~mm}$ culture dishes. After 10 days of incubation, the cells were fixed with $10 \%$ neutral buffer formalin fixative and stained with crystal violet staining solution (Beyotime, CHN). To count the numbers, colonies were photographed.

2.7. Transwell Invasive Assay. The invasive ability of OSCC cells was assessed by transwell invasive assay. Matrigel (BD
Biosciences, Franklin Lakes, NJ) was added into the upper chamber $(8 \mu \mathrm{m}$ pore size; Corning, Corning, NY) of 24 -well plates. The CAL-27/LVRU6MP and CAL-27/MMP9/shRNA cells $\left(1 \times 10^{6} / \mathrm{ml}\right)$ and SCC-15/LVRU6MP and SCC-15/MMP-9/shRNA cells $\left(5 \times 10^{6} / \mathrm{ml}\right)$ were, respectively, suspended in the serum-free medium containing $2 \%$ bovine serum albumin (BSA, VWR, Radnor, PA) and transferred to the upper chamber above Matrigel. Then, the routine medium containing 10\% FBS was added to the lower chamber. CAL-27/LVRU6MP and CAL-27/MMP-9/shRNA cells were cultured for $48 \mathrm{~h}$, and SCC-15/LVRU6MP and SCC-15/MMP-9/shRNA cells were cultured for $72 \mathrm{~h}$, respectively. The remaining cells that had not passed through Matrigel in the upper chamber were removed by cotton balls. Then, cells were fixed with $10 \%$ neutral buffer formalin fixative and stained with crystal violet staining solution. The pictures were taken under an inverted microscope for counting the numbers.

2.8. Zebrafish Maintenance. Wild-type (WT) Tuebingen (TU) strain of zebrafish was provided by State Key 
TABLE 1: The details of implantation.

\begin{tabular}{lccccccc}
\hline Cell line & $\begin{array}{c}\text { Fish } \\
\text { line }\end{array}$ & $\begin{array}{c}\text { Implantation } \\
\text { density }(/ \mathrm{ml})\end{array}$ & $\begin{array}{c}\text { Implantation } \\
\text { period }(\mathrm{hpf})\end{array}$ & $\begin{array}{c}\text { Number of } \\
\text { injected cells }\end{array}$ & $\begin{array}{c}\text { Number of xenografted } \\
\text { zebrafish }\end{array}$ & $\begin{array}{c}\text { Implantation } \\
\text { site }\end{array}$ & $\begin{array}{c}\text { Temperature } \\
\left({ }^{\circ} \mathrm{C}\right)\end{array}$ \\
\hline CAL-27/LVRU6MP & WT & $4.0 \times 10^{7}$ & 48 & $500-600$ & 120 & Yolk sac & 29 \\
CAL-27/MMP-9/shRNA & WT & $4.0 \times 10^{7}$ & 48 & $500-600$ & 120 & Yolk sac \\
SCC-15/LVRU6MP & WT & $4.0 \times 10^{7}$ & 48 & $500-600$ & 290 & Yolk sac \\
SCC-15/MMP-9/shRNA & WT & $4.0 \times 10^{7}$ & 48 & $500-600$ & 290 & Yolk sac & 29 \\
\hline
\end{tabular}

Laboratory of Membrane Biology, Institute of Zoology, University of Chinese Academy of Sciences, Chinese Academy of Sciences, and raised at $29^{\circ} \mathrm{C}$ under standard laboratory conditions. The embryos were collected and cultured in egg water containing $0.003 \%$ phenylthiourea (PTU, SigmaAldrich Corp). All in vivo experiments with zebrafish were repeated three times.

2.9. The Establishment of a Zebrafish OSCC Model. Before implantation, the CAL-27/LVRU6MP and CAL-27/MMP9/shRNA cells and SCC-15/LVRU6MP and SCC-15/MMP9/shRNA cells were, respectively, suspended in serum-free medium at a density of $4 \times 10^{7} / \mathrm{ml}$. At 48 hours postfertilization (hpf), dechorionated embryos were anesthetized with $0.4 \%$ tricaine (Sigma-Aldrich Corp) and placed in the petri dish covered with $1 \%$ low melting point agarose (SigmaAldrich Corp) in a certain order. Then, approximately $15 \mathrm{nl}$ cell suspension (500-600 cells) was injected into the top of the yolk sac using glass capillary needles (approximately an opening size of a single-cell diameter) by micromanipulator (MPPI-3, Applied Scientific Instrumentation, Eugene, OR) (see Table 1 for details). These injected embryos were immediately transferred into 12 -well plates (10 individuals per well) containing PTU egg water and maintained at $29^{\circ} \mathrm{C}$. After half an hour of implantation, we discarded the embryos that have tumor cells mistakenly injected into the vascular system.

2.10. Microscopy and Image Analysis. To investigate the role of the MMP-9 gene in OSCC cells, these injected zebrafish were anesthetized and photographed daily in 3\% methylcellulose using fluorescence stereoscopy (M205FA, Leica Microsystems, DE) from 0 to 6 days postinjection (dpi) (magnification, $\times 32, \times 63$ ). In brief, a red fluorescent focus indicated a metastatic tumor cell or lesion. The tumor metastatic distance was defined as the distance from the previous implantation location to the metastatic site [22]. Image J 1.0 (National Institutes of Health, Bethesda, MD) was used to measure the metastatic distance and count the number of metastatic cells or lesions. Additionally, the rate of metastasis was evaluated. It was calculated as follows: the metastasis rate $(\%)=($ the number of zebrafish with tumor metastasis / the initial number of xenografted zebrafish) $\times 100 \%$.

2.11. Statistical Analysis. Data were expressed as mean \pm standard deviations (SD). Statistical analysis was performed by the Student $t$-test for paired comparisons and chi-square test for sample rates using SPSS Statistics 25.0 (IBM, Armonk, NY). $P<0.05$ was deemed statistically significant.

\section{Results}

3.1. Establishment of a Knockdown Model of MMP-9 in OSCC Cells. To further study the role of the knockdown of MMP-9 in OSCC cells, we have constructed a mCherry-labeled OSCC cell knockdown model of MMP-9 (Figure 1(a)). The lentiviral vector information was provided in Supplemental Figure 1. Furthermore, RT-PCR and immunocytochemistry were performed to verify the decreasing expression of MMP-9. RT-PCR results showed that MMP-9 expression of the MMP-9/shRNA group was significantly reduced at the RNA level compared with the control (Figure 1(c)). Similarly, the level of MMP-9 protein in the MMP9/shRNA group was also significantly lower than that in the shRNA control group (Figure 1(b)).

3.2. Knockdown of MMP-9 Suppresses Proliferation, Colony Formation, and Invasion of OSCC Cells In Vitro. To further study the role of MMP-9 in OSCC cells in vitro, a series of experiments were performed. MTT assay results revealed that the knockdown of MMP-9 slowed down the proliferation of OSCC cells in a time-dependent manner (Figure 2). Additionally, colony formation assay results found that the knockdown of MMP-9 in OSCC cells inhibited the OSCC cell colony formation ability and reduced the size and number of colonies (Figure 3). Transwell invasive assay was used to investigate the invasive ability of OSCC cells. Matrigel acts as the basement membrane matrix, and cancer cells can only invade by destroying it. Compared with the control group, the knockdown of MMP-9 in OSCC cells significantly reduced the ability to invade (Figure 4 ). These data indicated that the knockdown of MMP-9 plays an inhibitor role in OSCC cell proliferation, colony formation, and invasion in vitro.

3.3. Knockdown of MMP-9 May Inhibit OSCC Cell Dissemination and Metastasis in a Zebrafish Xenograft Model. To further confirm the role of MMP-9 in OSCC cells in vivo, we established a novel human OSCC model in zebrafish by implanting tumor cells into the yolk sac of the embryo. The dissemination of OSCC cells out of the yolk sac is deemed as active metastasis and invasion because the yolk is considered an acellular and partly avascular environment that cannot support passive cell transport [22, 30]. At $3 \mathrm{dpi}$, some CAL-27/LVRU6MP cells escaping the yolk were observed in the pronephric region, trunk, and distal tail region (Figure 5(a)). But limited invasive CAL-27/MMP-9/shRNA cells were detected in the 
CAL-27

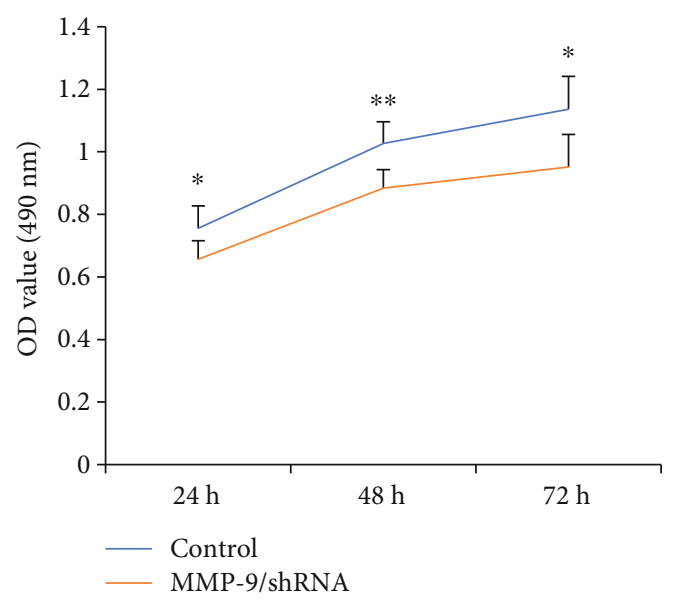

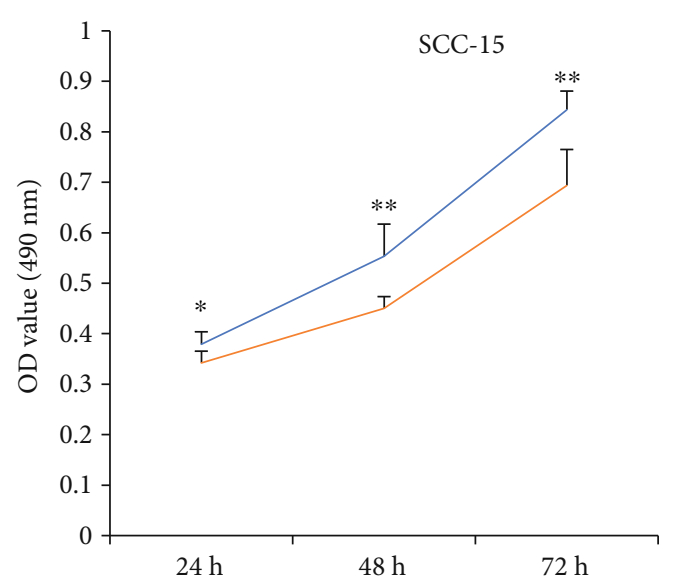

FIGURE 2: Knockdown of MMP-9 could slow down the growth of OSCC cells (CAL-27 and SCC-15 cells) in a time-dependent manner by MTT assay. ${ }^{*} P<0.05$ and ${ }^{* *} P<0.01$ as compared with the control.

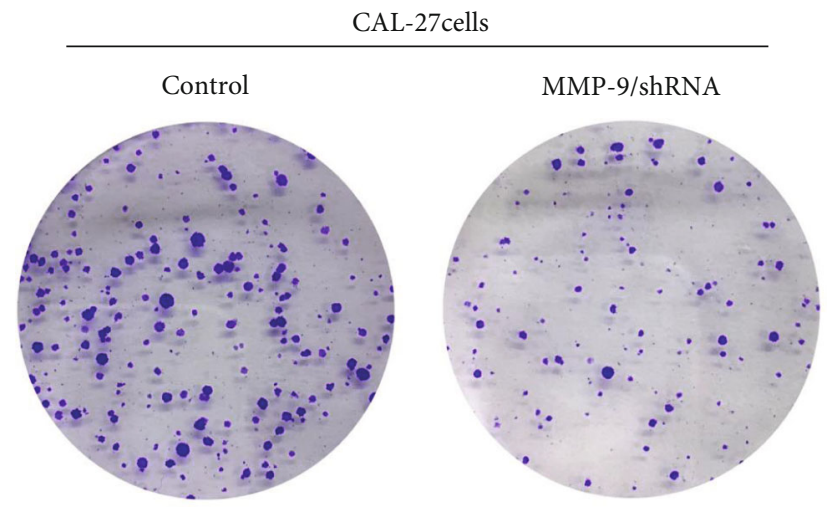

(a)

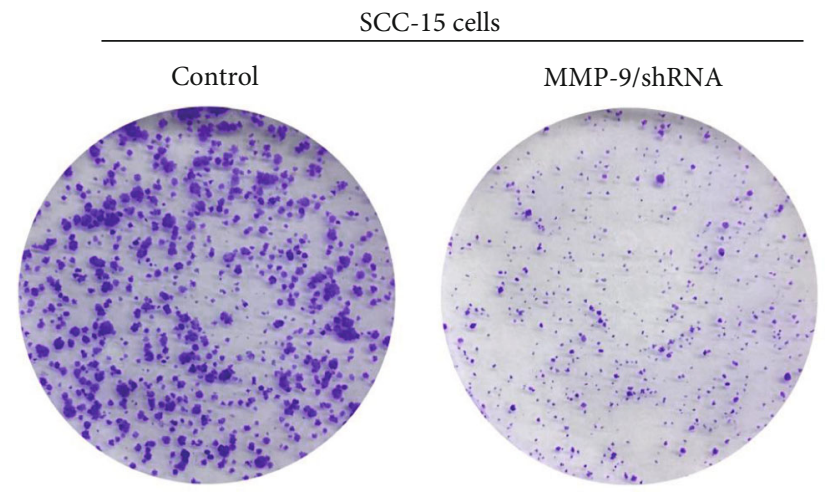

(b)
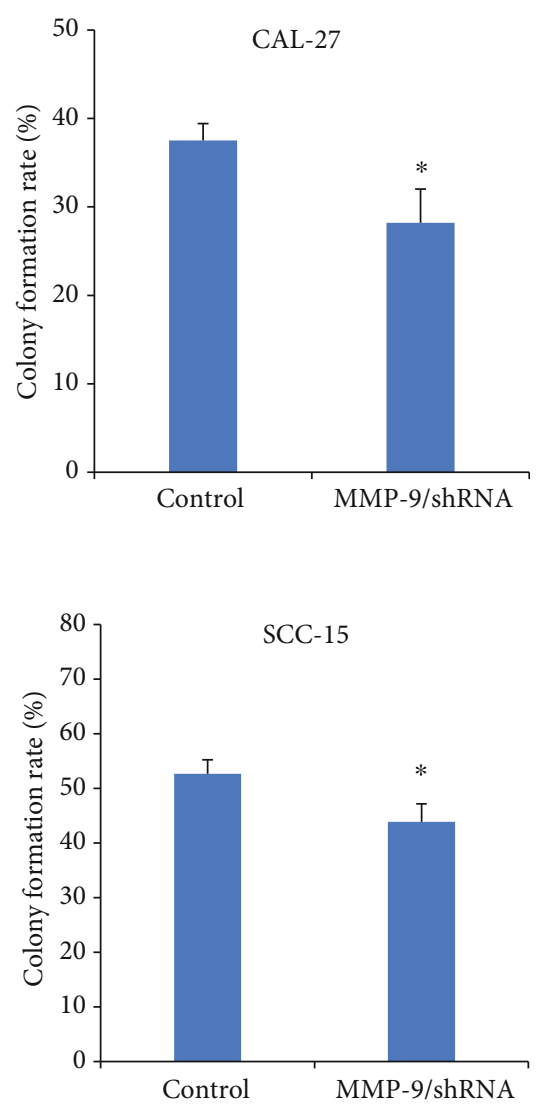

FIGURE 3: Knockdown of MMP-9 could inhibit the colony formation of OSCC cells (CAL-27 and SCC-15 cells) compared with the control. ${ }^{*} P<0.05$ as compared with the control.

trunk region and showed less metastatic distance (Figure 5(b)). In order to quantify the metastatic ability of different OSCC cell lines in the zebrafish body, ImageJ 1.0 software was applied to picture analysis. Data analysis showed that the knockdown of MMP-9 significantly reduced the average metastatic distance, maximal distance, and number of metastatic tumor cells or lesions in the zebrafish body at $3 \mathrm{dpi}$ (Figures 5(c)-5(e)). 

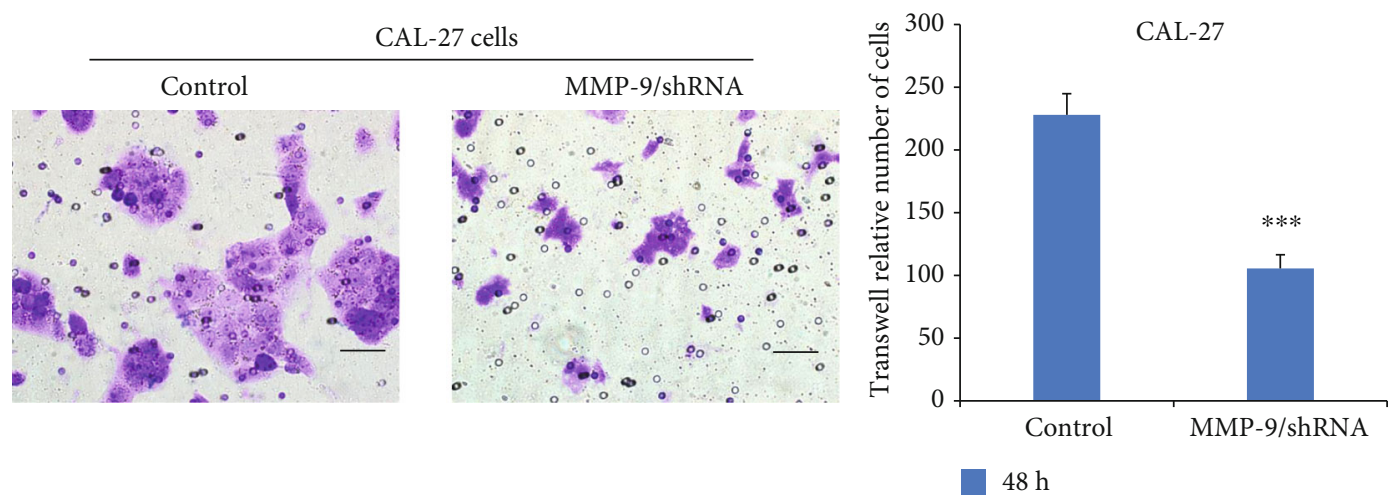

(a)
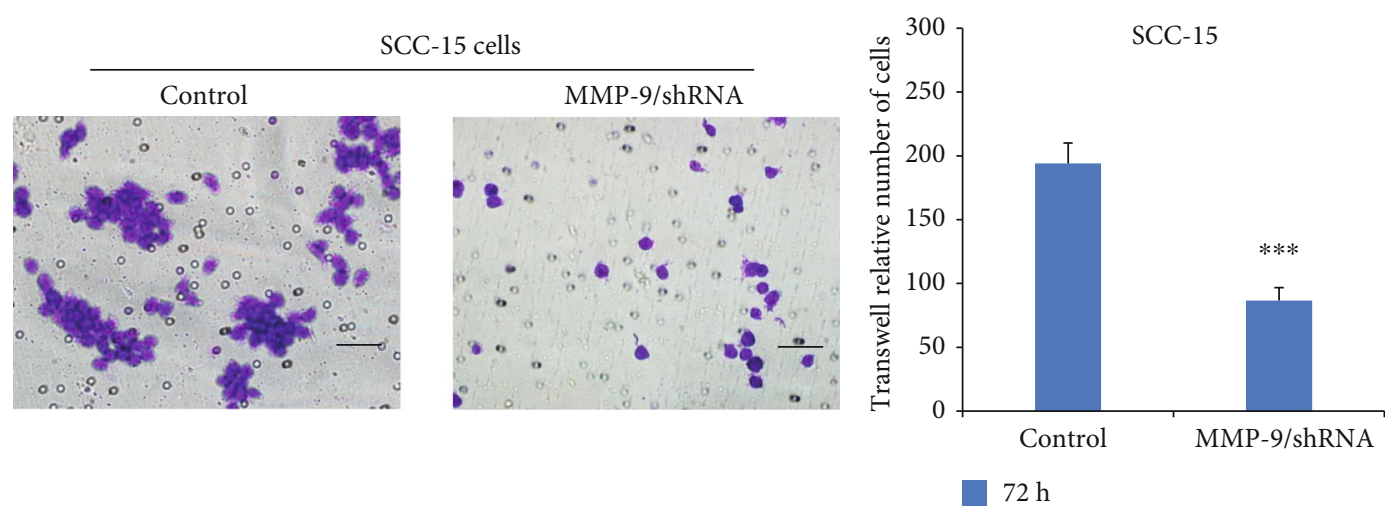

(b)

FIGURE 4: Knockdown of MMP-9 could decrease the invasive ability of OSCC cells (CAL-27 and SCC-15 cells) by transwell invasive assay (magnification, $\times 400$ ). ${ }^{* * *} P<0.001$ as compared with the control. Bar $=50 \mathrm{~mm}$.

To verify these results again, similar experiments were performed with SCC-15/LVRU6MP and SCC-15/MMP9/shRNA cells. At $5 \mathrm{dpi}$, we found that SCC-15/LVRU6MP cells had invaded the eye, pericardium, pronephric region, and distal tail region (Figure 6(a)). However, SCC15/MMP-9/shRNA cells were detected only in the pronephric region, and no cell invasion was detected in the distal tail region (Figure 6(b)). Quantification analysis revealed that the knockdown of MMP-9 decreased the average metastatic distance and number of metastatic tumor cells or lesions at 5 dpi. Meanwhile, decreasing metastasis rate from 3 dpi to 6 dpi in zebrafish was detected (Figures 6(c)-6(e)). Taken together, the knockdown of MMP-9 in OSCC cells may attenuate the invasive and metastasis ability of OSCC cells in vivo. These results in the zebrafish xenograft model are consistent with the behavior in vitro.

\section{Discussion}

Cancer cell metastasis has complicated steps, and the detected metastatic tumor mass implies the final step of metastasis in the clinical setting [21]. OSCC is one of most common cancers in humans [31], and its initial lymph node metastasis is common and difficult to be observed at the early stages, possibly due to opaque tissues in human and mice. Importantly, the processes of tumor growth and metastasis in a mouse OSCC model were generally studied after mice are sacrificed. Furthermore, the nude mouse OSCC model has limited utility for large screening of anticancer agents due to many limitations including slow solid tumor growth, high breeding cost, large housing space, and much time for examining mice. However, the early translucent zebrafish embryo develops fast, allows live image in vivo, and absorbs compounds in water, and it has been reported that xenografts are not rejected owing to the immature immune system [32]. Therefore, the zebrafish embryo can be used as an ideal tool to observe tumor proliferation, angiogenesis, and metastasis in a real-time manner. In short, after implanting cancer cells into the yolk, the tumor progression includes proliferating, evading attack of immune systems, promoting angiogenesis, intravasating into the circulation, migrating through vasculature, extravasating into the zebrafish body (frequently the head and distal tail region), and proliferating at the new location [33]. From the perspective of tumor metastasis, suppressing any of these steps might prevent cancer cell metastasis. In our study, we established here a novel human OSCC model in zebrafish to investigate the role of the knockdown of MMP-9 in OSCC cells. Additionally, this model may play an important role in evaluating real-time therapeutic effect and high-throughput screening of antioral cancer agents in the future.

In previous studies, MMP-9 has been reported to destroy collagen IV, the main ingredient of basement membranes, and participate in remodeling, angiogenesis, and cancer cell 


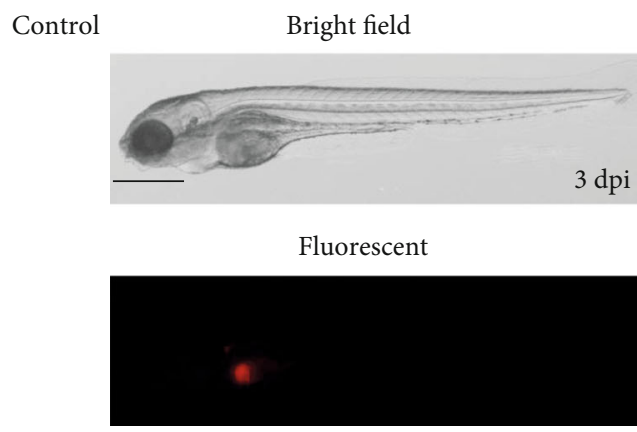

MMP-9/shRNA

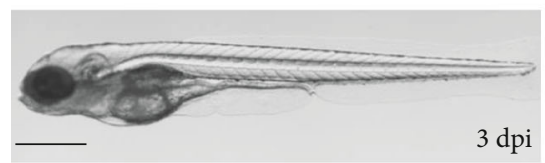

Fluorescent
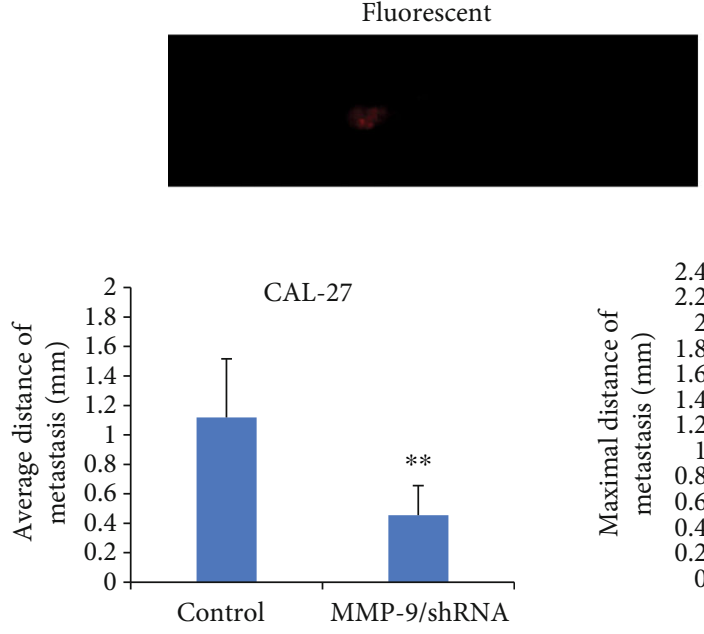

(c)

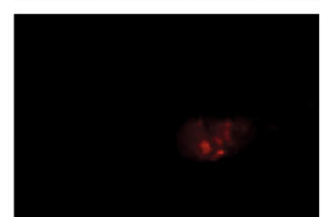

(b)
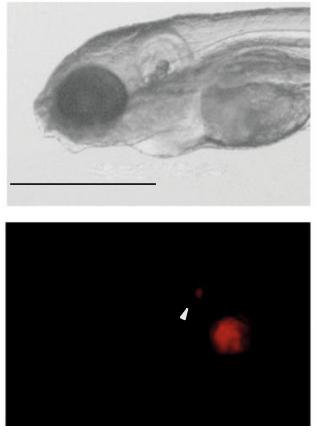

(a)
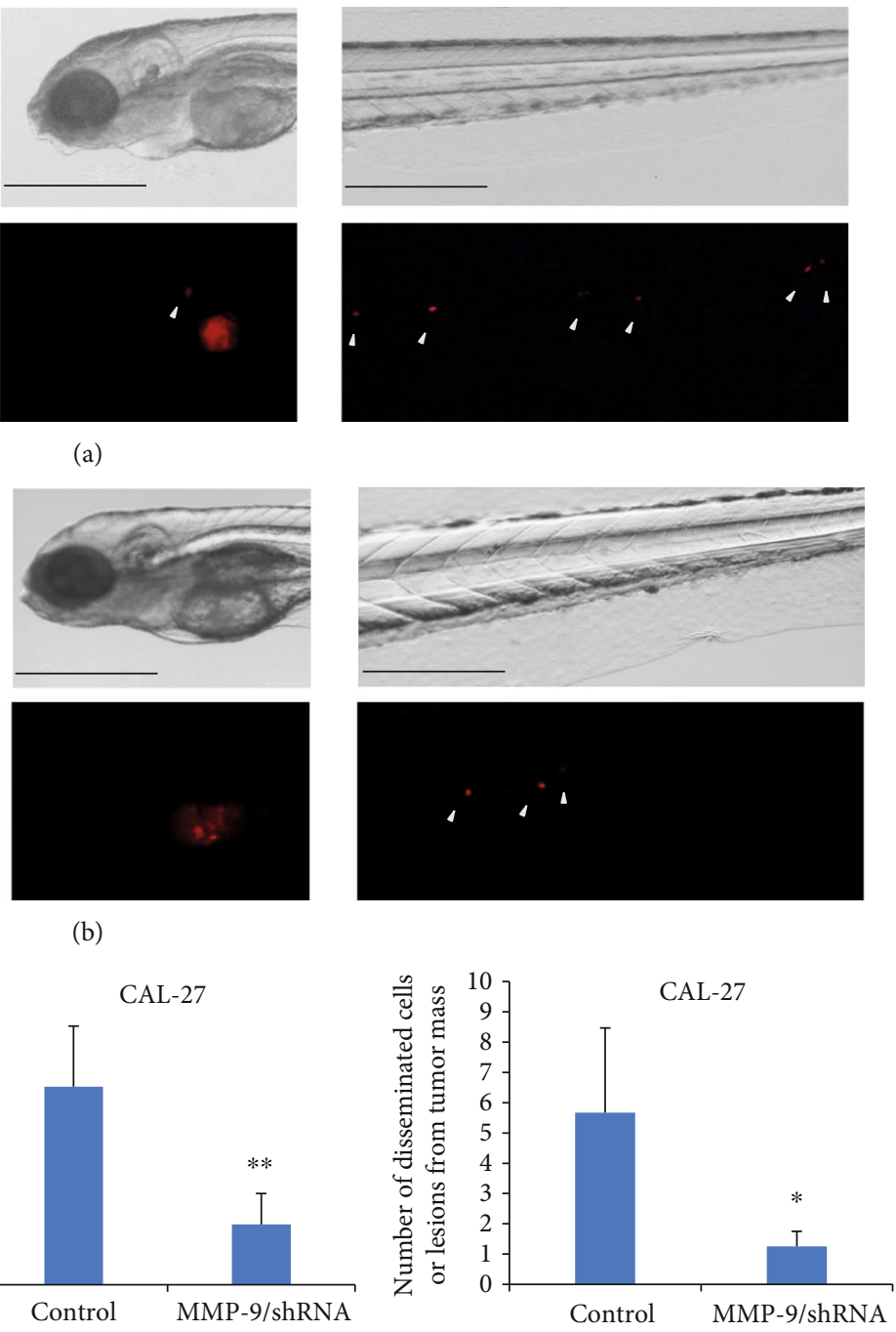

(e)

FIGURE 5: Knockdown of MMP-9 decreased the metastatic distance and number of CAL-27 cells in the zebrafish xenograft model. A red fluorescent cluster indicated a tumor mass, and the foci marked by white arrows indicated the metastatic tumor cells or lesions that have escaped from the yolk sac. $(\mathrm{a}, \mathrm{b})$ The bright-field and fluorescent pictures of whole-body, head, distal tail regions of zebrafish injected with CAL-27/LVRU6MP and CAL-27/MMP-9/shRNA cells at 3 dpi $(n=120$; magnification, $\times 32, \times 63)$. (c, e) Knockdown of MMP-9 reduced the invasive average distance, maximal distance, and number of disseminated cells or lesions at 3 dpi. ${ }^{*} P<0.05$ and ${ }^{* *} P<0.01$ as compared with the control. $\mathrm{Bar}=0.5 \mathrm{~mm}$.

metastasis in some solid tumors [34]. In brief, MMP-9 may act as a tumor promoter in many solid tumors [10-13]. In our study, we found similar results. The knockdown of MMP-9 may effectively inhibit OSCC cell metastasis and invasion in xenografted zebrafish. These evidences confirmed that the role of MMP-9 in OSCC cells is consistent with that in series of cancers and relevant clinical researches [15-18].

As previously described, angiogenesis is an important step of cancer metastasis and facilitates distant metastasis. Studies have shown that tumor-induced angiogenesis was detected after human melanoma cells were injected into the zebrafish embryo yolk and hindbrain ventricle [35]. Moreover, the formation of angiogenic sprouts and neovascularization could be observed by implanting mouse melanoma cells or colon cancer cells into the embryo perivitelline space.
And this process was suppressed by SU5416 (vascular endothelial growth factor receptor 2 (VEGFR2) inhibitor) [36]. In other studies, Ewing sarcoma cells have also been reported to induce sprouting of the subintestinal vein (SIV) and growth in the zebrafish xenograft model [37]. Thus, we hypothesize that human OSCC cells might invade into the fish body by stimulating sprouting of the SIV. However, the underlying mechanisms that reduction of MMP-9 expression in OSCC cells may inhibit this process remains unclear in the zebrafish model and needs to be further studied.

\section{Conclusions}

In summary, the knockdown of MMP-9 in OSCC cells might inhibit tumor cell metastasis and invasion and reduce the 
Control

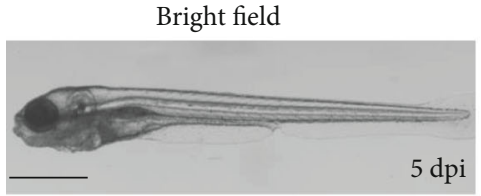

Fluorescent
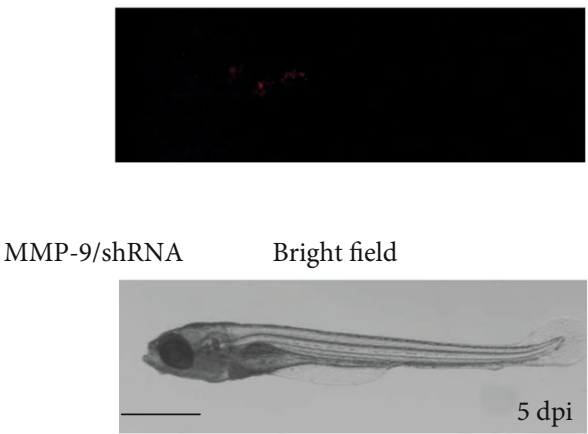

Fluorescent

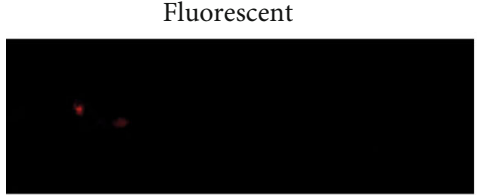

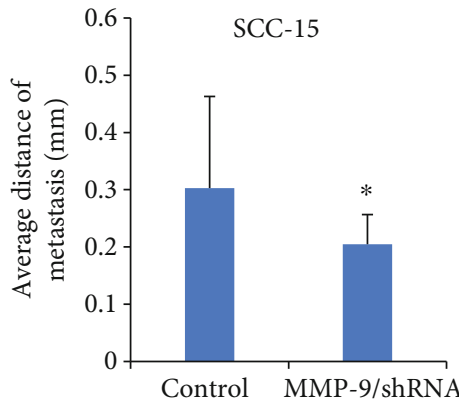

(c)

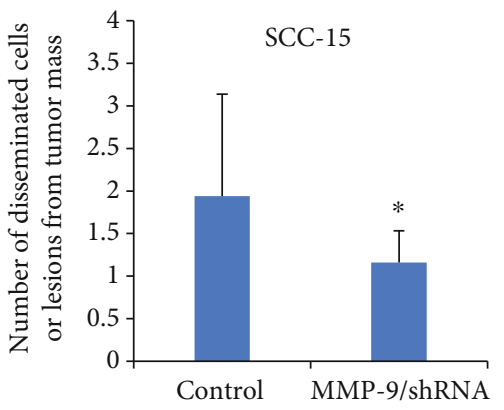

(d)

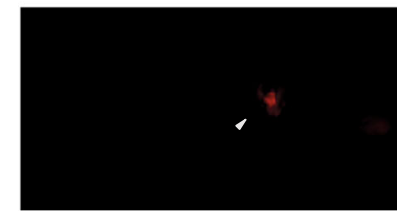

(b)
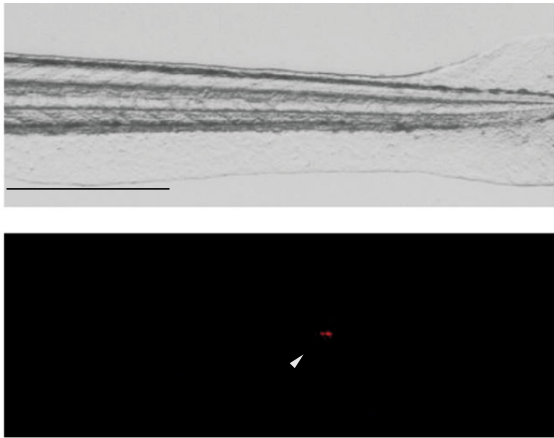

(a)
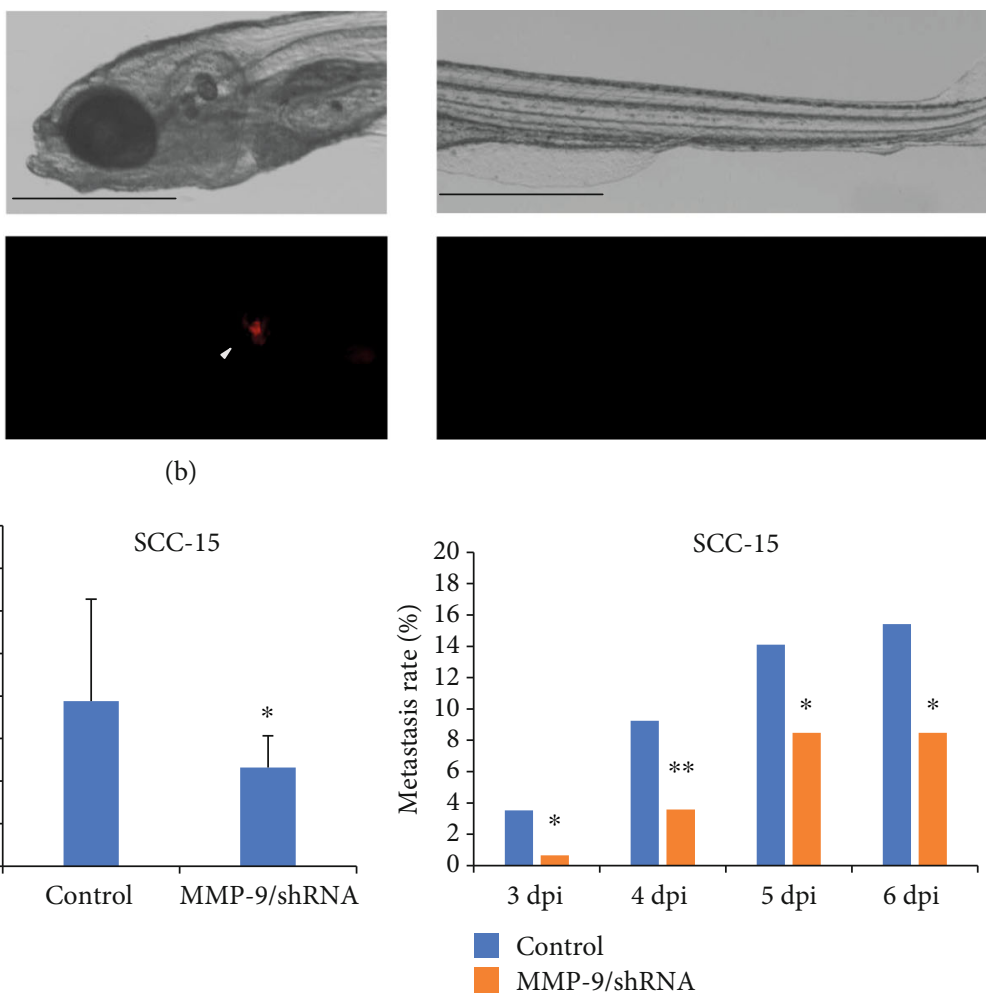

(e)

FIGURE 6: Knockdown of MMP-9 reduced the metastatic distance and number of SCC-15 cells and decreased the metastasis rate in the zebrafish xenograft model. $(\mathrm{a}, \mathrm{b})$ The bright-field and fluorescent pictures of whole-body, head, distal tail regions of zebrafish injected with SCC-15/LVRU6MP $(n=230$; magnification, $\times 32, \times 63)$ and SCC-15/MMP-9/shRNA cells at 5 dpi $(n=290$; magnification, $\times 32, \times 63)$. (c, e) Knockdown of MMP-9 decreased the invasive average distance and number of disseminated cells or lesions at 5 dpi and inhibited the metastasis rate from 3 dpi to 6 dpi. ${ }^{*} P<0.05$ and ${ }^{* *} P<0.01$ as compared with the control. Bar $=0.5 \mathrm{~mm}$.

metastasis rate of xenografted zebrafish. It was confirmed that MMP-9 may play a key role in OSCC cell metastasis and invasion in vivo and in vitro. These results imply that the MMP-9 gene may act as a promising therapeutic target in OSCCs. But underlying molecular mechanisms need to be further investigated in the future study.

\section{Abbreviations}

MMP-9: Matrix metallopeptidase 9

MMPs: Matrix metalloproteinases

OSCC: Oral squamous cell carcinoma

ECM: $\quad$ Extracellular matrix
EGFR: Epidermal growth factor receptor

FBS: $\quad$ Fetal bovine serum

DMSO: Dimethyl sulfoxide

OD: Optical density

BSA: $\quad$ Bovine serum albumin

PTU: $\quad$ Phenylthiourea

hpf: $\quad$ Hours postfertilization

dpi: Days postinjection

SIV: $\quad$ Subintestinal vein

VEGFR2: Vascular endothelial growth factor receptor 2

DAB: Diaminobenzidine.

WT: Wild-type.

TU: Tuebingen. 


\section{Data Availability}

The data used to support the findings of this study are included within the article.

\section{Ethical Approval}

The authors declare that all the experimental procedures were in accordance with the institutional guidelines of the Animal Care Committee at the Institute of Zoology, University of Chinese Academy of Sciences, Chinese Academy of Sciences (Permission Number: IOZ-13048).

\section{Conflicts of Interest}

The authors declare that they have no conflicts of interest.

\section{Authors' Contributions}

Jinlin Wen completed the experimental design, performed the experiments, and wrote the manuscript. Linwei Li and Guihua Kang contributed to experiments in vivo. Panpan Yin, Feng Gao, and Yanlin $\mathrm{Wu}$ contributed to experiments in vitro. Guozhu Ning, Yu Cao, and Ying Su contributed technical or material support. Xinyan Zhang put forward the experimental idea, supervised the research, and revised the manuscript.

\section{Acknowledgments}

We want to thank Professor Qiang Wang from State Key Laboratory of Membrane Biology, Institute of Zoology, University of Chinese Academy of Sciences, Chinese Academy of Sciences, for providing the technical help and guidance in zebrafish experiments. This research was supported by the National Nature Science Foundation of China (81772868) and a grant from Beijing Municipal Natural Science Foundation (7202057).

\section{Supplementary Materials}

Supplemental Figure 1: the shRNA expression clone datasheet. (a) The vector information. (b) The shRNA target sequences. Sequence 33 was selected for subsequent lentivirus packaging and transfection. Supplemental Figure 2: knockdown of MMP-9 in CAL-27 cells could slightly inhibit the metastasis rate from $3 \mathrm{dpi}$ to $5 \mathrm{dpi}$, but the results were not statistically significant. ns: no significance. (Supplementary Materials)

\section{References}

[1] J. Ferlay, I. Soerjomataram, R. Dikshit et al., "Cancer incidence and mortality worldwide: sources, methods and major patterns in GLOBOCAN 2012," International Journal of Cancer, vol. 136, no. 5, pp. E359-E386, 2015.

[2] S. N. Rogers, J. S. Brown, J. A. Woolgar et al., "Survival following primary surgery for oral cancer," Oral Oncology, vol. 45, no. 3, pp. 201-211, 2009.
[3] J. Noguti, C. F. de Moura, G. P. de Jesus et al., "Metastasis from oral cancer: an overview," Cancer Genomics Proteomics, vol. 9, no. 5, pp. 329-335, 2012.

[4] C. Walker, E. Mojares, and A. Del Rio Hernandez, "Role of extracellular matrix in development and cancer progression," International Journal of Molecular Sciences, vol. 19, no. 10, p. $3028,2018$.

[5] T. T. Chang, D. Thakar, and V. M. Weaver, "Force-dependent breaching of the basement membrane," Matrix Biology, vol. 57-58, pp. 178-189, 2017.

[6] Y. Chen, T. Jiang, A. Mao, and J. Xu, "Esophageal cancer stem cells express PLGF to increase cancer invasion through MMP9 activation," Tumor Biology, vol. 35, no. 12, pp. 12749-12755, 2014.

[7] Z. H. Jia, Y. Jia, F. J. Guo, J. Chen, X. W. Zhang, and M. H. Cui, "Phosphorylation of STAT3 at Tyr705 regulates MMP-9 production in epithelial ovarian cancer," PLoS One, vol. 12, no. 8, p. e0183622, 2017.

[8] G. Bergers, R. Brekken, G. McMahon et al., "Matrix metalloproteinase-9 triggers the angiogenic switch during carcinogenesis," Nature Cell Biology, vol. 2, no. 10, pp. 737-744, 2000.

[9] E. I. Deryugina and J. P. Quigley, "Matrix metalloproteinases and tumor metastasis," Cancer and Metastasis Reviews, vol. 25, no. 1, pp. 9-34, 2006.

[10] P. Puolakkainen, A. Koski, S. Vainionpää et al., “Anti-inflammatory macrophages activate invasion in pancreatic adenocarcinoma by increasing the MMP9 and ADAM8 expression," Medical Oncology, vol. 31, no. 3, p. 884, 2014.

[11] F. Lin, X. Chengyao, L. Qingchang, D. Qianze, W. Enhua, and W. Yan, "CRKL promotes lung cancer cell invasion through ERK-MMP9 pathway," Molecular Carcinogenesis, vol. 54, no. S1, pp. E35-E44, 2015.

[12] Q. Yang, M. Ji, H. Guan, B. Shi, and P. Hou, "Shikonin inhibits thyroid cancer cell growth and invasiveness through targeting major signaling pathways," Journal of Clinical Endocrinology \& Metabolism, vol. 98, no. 12, pp. E1909E1917, 2013.

[13] H. Zhang, C. Hao, Y. Wang et al., "Sohlh2 inhibits human ovarian cancer cell invasion and metastasis by transcriptional inactivation of MMP9," Molecular Carcinogenesis, vol. 55, no. 7, pp. 1127-1137, 2016.

[14] A. Pujada, L. Walter, A. Patel et al., "Matrix metalloproteinase MMP9 maintains epithelial barrier function and preserves mucosal lining in colitis associated cancer," Oncotarget, vol. 8, no. 55, pp. 94650-94665, 2017.

[15] L. S. Monteiro, M. L. Delgado, S. Ricardo et al., "Prognostic significance of CD44v6, p 63, podoplanin and MMP-9 in oral squamous cell carcinomas," Oral Diseases, vol. 22, no. 4, pp. 303-312, 2016.

[16] R. N. F. Silva, L. B. Dallarmi, A. K. C. Araujo et al., "Immunohistochemical analysis of neutrophils, interleukin-17, matrix metalloproteinase- 9 , and neoformed vessels in oral squamous cell carcinoma," Journal of Oral Pathology \& Medicine, vol. 47, no. 9, pp. 856-863, 2018.

[17] N. A. Ghallab and O. G. Shaker, "Serum and salivary levels of chemerin and MMP-9 in oral squamous cell carcinoma and oral premalignant lesions," Clinical Oral Investigations, vol. 21, no. 3, pp. 937-947, 2016.

[18] A. Peisker, G. F. Raschke, M. D. Fahmy et al., "Salivary MMP-9 in the detection of oral squamous cell carcinoma," Medicina 
Oral Patología Oral y Cirugia Bucal, vol. 22, no. 3, pp. e270e275, 2017.

[19] R. White, K. Rose, and L. Zon, "Zebrafish cancer: the state of the art and the path forward," Nature Reviews Cancer, vol. 13, no. 9, pp. 624-636, 2013.

[20] M. C. Mione and N. S. Trede, "The zebrafish as a model for cancer," Disease Models \& Mechanisms, vol. 3, no. 9-10, pp. 517-523, 2010.

[21] C. Liu, Y.Zhang, S. Lim et al., "A zebrafish model discovers a novel mechanism of stromal fibroblast-mediated cancer metastasis," Clinical Cancer Research, vol. 23, no. 16, pp. 4769-4779, 2017.

[22] W. van der Ent, C. Burrello, A. F. A. S. Teunisse et al., "Modeling of human uveal melanoma in zebrafish xenograft embryos," Investigative Ophthalmology \& Visual Science, vol. 55, no. 10, p. 6612, 2014.

[23] K. C. de Sousa Pontes, A. Groenewoud, J. Cao, L. M. S. Ataide, E. Snaar-Jagalska, and M. J. Jager, "Evaluation of (fli:GFP)CasperZebrafish embryos as a model for human conjunctival melanoma," Investigative Ophthalmology \& Visual Science, vol. 58, no. 14, p. 6065, 2017.

[24] D. Li, X.-P. Li, H.-X. Wang et al., "VEGF induces angiogenesis in a zebrafish embryo glioma model established by transplantation of human glioma cells," Oncology Reports, vol. 28, no. 3, pp. 937-942, 2012.

[25] L. Mercatali, F. La Manna, A. Groenewoud et al., "Development of a patient-derived xenograft (PDX) of breast cancer bone metastasis in a zebrafish model," International Journal of Molecular Sciences, vol. 17, no. 8, p. 1375, 2016.

[26] L. M. J. Lee, E. A. Seftor, G. Bonde, R. A. Cornell, and M. J. C. Hendrix, "The fate of human malignant melanoma cells transplanted into zebrafish embryos: assessment of migration and cell division in the absence of tumor formation," Developmental Dynamics, vol. 233, no. 4, pp. 15601570, 2005.

[27] C. Liverani, F. la Manna, A. Groenewoud et al., "Innovative approaches to establish and characterize primary cultures: an ex vivo 3D system and the zebrafish model," Annals of Oncology, vol. 27, p. iv117, 2016.

[28] J. T. Baeten and J. L. O. de Jong, "Genetic models of leukemia in zebrafish," Frontiers in Cell and Developmental Biology, vol. 6, 2018.

[29] L. I. Zon and R. T. Peterson, “_In vivo_drug discovery in the zebrafish," Nature Reviews Drug Discovery, vol. 4, no. 1, article BFnrd1606, pp. 35-44, 2005.

[30] J. Wertman, C. J. Veinotte, G. Dellaire, and J. N. Berman, “The zebrafish xenograft platform: evolution of a novel cancer model and preclinical screening tool," Advances in Experimental Medicine and Biology, vol. 916, 2016.

[31] X. Zhang, L. Zhang, X. Tan et al., "Systematic analysis of genes involved in oral cancer metastasis to lymph nodes," Cellular \& Molecular Biology Letters, vol. 23, no. 1, 2018.

[32] J. Wang, Z. Cao, X. M. Zhang et al., "Novel mechanism of macrophage-mediated metastasis revealed in a zebrafish model of tumor development," Cancer Research, vol. 75, no. 2, pp. 306-315, 2015.

[33] A. B. Mohseny and P. C. W. Hogendoorn, "Zebrafish as a model for human osteosarcoma," Advances in Experimental Medicine and Biology, vol. 804, 2014.
[34] M. Egeblad and Z. Werb, "New functions for the matrix metalloproteinases in cancer progression," Nature Reviews Cancer, vol. 2, no. 3, pp. 161-174, 2002.

[35] M. Haldi, C. Ton, W. L. Seng, and P. McGrath, "Human melanoma cells transplanted into zebrafish proliferate, migrate, produce melanin, form masses and stimulate angiogenesis in zebrafish," Angiogenesis, vol. 9, no. 3, article 9040, pp. 139$151,2006$.

[36] C. Zhao, X. Wang, Y. Zhao et al., "A novel xenograft model in zebrafish for high-resolution investigating dynamics of neovascularization in tumors," PLoS One, vol. 6, no. 7, p. e21768, 2011.

[37] W. van der Ent, A. G. Jochemsen, A. F. A. S. Teunisse et al., "Ewing sarcoma inhibition by disruption of EWSR1-FLI1 transcriptional activity and reactivation of p53," The Journal of Pathology, vol. 233, no. 4, pp. 415-424, 2014. 